\title{
SCHUR HOMOTOPY OF THE SIMPLEST GROUP
}

\author{
BY CHARLES H. GIFFEN ${ }^{1}$
}

Communicated by Hyman Bass, February 7, 1974

Let $G$ be a perfect group; that is, $G=[G, G]$, the commutator subgroup of $G$, or equivalently $H_{1}(G ; Z)=0$. In [1], Quillen has shown that for any space $X$ with $\pi_{1} X$ perfect, there are a simply-connected space $X^{+}$unique up to homotopy type and a map $j: X \rightarrow X^{+}$such that $j_{*}: H_{*}(X ; C) \rightarrow$ $H_{*}\left(X^{+} ; C\right)$ is an isomorphism for all coefficient groups $C$; moreover, any two maps inducing the same isomorphism $H_{*}(X ; Z) \rightarrow H_{*}\left(X^{+} ; Z\right)$ are homotopic. Alternatively, $j: X \rightarrow X^{+}$has the characterizing property that for every map $g: X \rightarrow Z$, where $Z$ is simply connected, there is a unique homotopy class of maps $g^{+}: X^{+} \rightarrow Z$ such that $g \simeq j g^{+}$. Furthermore, an $X^{+}$may be obtained from $X$ by attaching only 2-cells and 3-cells.

If now $f: X \rightarrow Y$ has the property that $G=f_{*} \pi_{1} X$ is a normal subgroup of $\pi_{1} Y$, then $G$ is also perfect, and define $Y_{G}^{+}$so that the following diagram is a homotopy cofiber pushout.

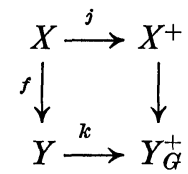

Take, e.g., $Y_{G}^{+}$homotopy equivalent to the double mapping cylinder of $Y \leftarrow X \rightarrow X^{+}$. Then $\pi_{1} Y_{G}^{+}=\pi_{1} Y / G$ and $k_{*}: H_{*}(Y ; E) \rightarrow H_{*}\left(Y_{G}^{+} ; E\right)$ is an isomorphism for every $Z\left[\pi_{1} Y \mid G\right]$-module $E$. The notation $Y_{G}^{+}$for the above pushout suggests what is actually a fact-namely that $Y_{G}^{+}$and $k$ are homotopy unique, depending only upon the perfect normal subgroup $G \subset \pi_{1} Y$ and not upon the choice of $X$ and $f$. Moreover, given the perfect normal subgroup $G$ of $\pi_{1} Y$, appropriate $f: X \rightarrow Y$ exist to define $Y_{G}^{+}$; in fact, $Y_{G}^{+}$may be obtained from $Y$ by attaching only 2-cells and 3-cells. Also, $k: Y \rightarrow Y_{G}^{+}$has a characterizing universal mapping property similar to the one described above for $j: X \rightarrow X^{+}$.

If $\phi: G \rightarrow A$ is a homomorphism of groups with $G$ perfect and $\phi G$ normal in $A$ (of course, $\phi G$ is also perfect), then there is the space $(B A)_{\phi}^{+}$, where

AMS (MOS) subject classifications (1970). Primary 18H10, 20D05, 55E40; Secondary $18 \mathrm{~F} 25,20 \mathrm{~J} 10,55 \mathrm{~F} 40$.

Key words and phrases. Perfect group, classifying space, Schur multiplier, homotopy group, binary icosahedral group, dodecahedral space, 3-sphere, Bockstein.

${ }^{1}$ Research supported by the National Science Foundation. 
$B A$ denotes a classifying space for the group $A$. If the homomorphism $\phi$ is understood, then we may also write $(B A)_{G}^{+}$for $(B A)_{\phi}^{+}$. The homotopy of $(B A)_{\phi G}^{+}$has proved interesting for certain $\phi: G \rightarrow A$; for example,

$\pi_{*}\left(K_{0} R \times(B G L(R))_{E(R)}^{+}\right) \cong K_{*} R$, Quillen's higher algebraic $k$-theory, $\pi_{*}\left(Z \times\left(B \Sigma_{\infty}\right)_{A_{\infty}}^{+}\right) \cong \pi_{*}^{S}, \quad$ stable homotopy of spheres (see [2]).

DEFINITION. If $G$ is any perfect group, then define the Schur space of $G$ to be

$$
\operatorname{Sch} G=(B \text { Aut } G)_{G}^{+},
$$

where $G \rightarrow$ Aut $G$ is the canonical homomorphism $g \mapsto$ inner automorphism induced by $g \in G$. The Schur homotopy groups of $G$ are defined to be the groups

$$
\mathrm{Sch}_{*} G=\pi_{*} \operatorname{Sch} G
$$

The terminology just introduced is to some extent justified by the following, which follows directly from Quillen [1].

Proposition. If $G$ is a perfect group, then

$$
\begin{aligned}
& \operatorname{Sch}_{1} G \cong \text { Out } G=\text { Aut } G /(\text { inner automorphisms }) \\
& \operatorname{Sch}_{2} G \cong H_{2}(G / \text { center } ; Z)=M(G / \text { center }) \\
&=\text { Schur multiplier of } G / \text { center }, \\
& \operatorname{Sch}_{3} G \cong H_{3}(\widetilde{G} ; Z)
\end{aligned}
$$

where $\tilde{G}$ denotes the largest perfect group such that there is a central extension $0 \rightarrow M \rightarrow \widetilde{G} \rightarrow G \rightarrow 1$. In general,

$$
\operatorname{Sch}_{n} G \cong \operatorname{Sch}_{n} \tilde{G} \cong \pi_{n}(B G)^{+} \text {for } n \geqq 3 .
$$
G.)

(Note that $M=M(G) \cong H_{2}(G ; Z) \cong \pi_{2}(B G)^{+}$is the Schur multiplier of

Understanding or computing higher Schur homotopy groups can be quite a problem. The only concrete examples are typified by $\operatorname{Sch} A_{\infty}=$ $\left(B \Sigma_{\infty}\right)_{A_{\infty}}^{+}$with homotopy groups $\operatorname{Sch}_{*} A_{\infty} \cong \pi_{*}^{S}$. Particularly intractible seem to be the Schur homotopy groups of finite perfect groups for which next to nothing is known. One problem in this situation is that there is no hope of utilizing infinite loop space (or generalized cohomology theoretic) techniques. In a paper to be published elsewhere, we intend to discuss some more or less systematic computational techniques which yield partial information on the Schur homotopy of finite groups.

For the remainder of this paper, we discuss in detail the Schur homotopy of the simplest possible perfect group $A_{5}$. We are fortunate that some 
rather ad hoc methods may be used to obtain a reasonably good description of $\mathrm{Sch}_{*} A_{5}$. From the Proposition above, we have immediately that $\operatorname{Sch}_{1} A_{5} \cong Z_{2}, \operatorname{Sch}_{2} A_{5} \cong Z_{2}$, and $\operatorname{Sch}_{3} A_{5} \cong H_{3}(\Delta ; Z) \cong Z_{120}$ where $\tilde{A}_{5} \cong \Delta$ is the binary icosahedral group of order 120 , presented by

$$
\Delta=g p\left\{x, y: x^{5}=y^{3}=(x y)^{2}\right\}
$$

(see, for example, Coxeter-Moser [3, §6.5]). The center of $\Delta$ is of order 2 generated by $z=(x y)^{2}$, and setting $z=1$ gives a classical presentation for $A_{5}$. There is a faithful, irreducible representation $\Delta \hookrightarrow S U(2) \cong S^{3}$ whose homogeneous space $\Sigma=S^{3} / \Delta$ is the Poincaré dodecahedral space. Now $\Sigma$ has the same homology as the 3-sphere $S^{3}$. In fact, identifying the complement of a nice open 3-cell in $\Sigma$ to a point gives a degree 1 map $j: \Sigma \rightarrow S^{3}$ such that $j_{*}: H_{*}(\Sigma ; C) \rightarrow H_{*}\left(S^{3} ; C\right)$ is an isomorphism for all $C$; in other words, $S^{3} \simeq \Sigma^{+}$.

The representation $\Delta \hookrightarrow S^{3}$ induces a map on classifying spaces $p: B \Delta \rightarrow$ $B S^{3}$, which may be taken to be a fibration with fiber $\Sigma$; equivalently, the total space $E$ of the principal $S^{3}$ bundle over $B \Delta$ induced by $p$ is canonically homotopy equivalent to $\Sigma$. But since $B S^{3}$ is simply connected, $p$ factors homotopy uniquely through $(B \Delta)^{+}$by a map $p^{+}:(B \Delta)^{+} \rightarrow B S^{3}$ with $p \simeq j p^{+}$. Hence, the principal $S^{3}$ bundle over $B \Delta$ mentioned above is induced by $j: B \Delta \rightarrow(B \Delta)^{+}$from the principal $S^{3}$ bundle over $(B \Delta)^{+}$induced by $p^{+}$. Since $j_{*}: H_{*}(B \Delta ; C) \rightarrow H_{*}\left((B \Delta)^{+} ; C\right)$ is an isomorphism for all $C$, then, by the comparison theorem for the Serre spectral sequence, and since $S^{3}$ and $(B \Delta)^{+}$are simply connected, the total space of the bundle over $(B \Delta)^{+}$ is just $E^{+} \simeq \Sigma^{+} \simeq S^{3}$. The inclusion of fiber $S^{3} \hookrightarrow E \simeq \Sigma$ has degree \pm 120 , and thus so does the inclusion of fiber $S^{3} \subseteq E^{+} \simeq S^{3}$. In other words, we have a homotopy fibration

$$
S^{3} \stackrel{120}{\longrightarrow} S^{3} \longrightarrow(B \Delta)^{+} .
$$

Taking homotopy groups yields an exact couple of "Bockstein" type,

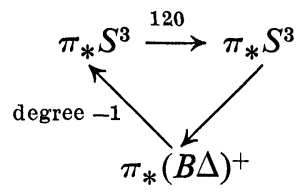

with $E_{*}^{1}=\pi_{*}(B \Delta)^{+}$. Also, $\pi_{n}(B \Delta)^{+} \cong \operatorname{Sch}_{n} A_{5}$ for $n \geqq 3$. Thus $\operatorname{Sch}_{4} A_{5} \cong Z_{2}$, $\mathrm{Sch}_{5} A_{5}$ is of order 4 , and $\mathrm{Sch}_{6} A_{5}$ is an extension of $Z_{12}$ by $Z_{2}$. In general, $\mathrm{Sch}_{*} A_{5}$ has no $p$-torsion for primes $p>5$.

Deep results of James (for $p=2$ ) and Toda (for $p$ odd) show that $\pi_{*} S^{3}$ has no elements of order $p^{3}$ for any prime $p$ (see [4], [5]). Although there are plenty of elements of order 4 , there is no known element of $\pi_{*} S^{3}$ of 
order $p^{2}$ for $p$ odd. It follows for $p=2$ and perhaps also for $p=3,5$ that there are short exact sequences for $n>4$,

$$
0 \rightarrow \pi_{n} S^{3} \otimes Q_{p} \rightarrow \operatorname{Sch}_{n} A_{5} \otimes Q_{p} \rightarrow \pi_{n-1} S^{3} \otimes Q_{p} \rightarrow 0,
$$

where $\boldsymbol{Q}_{p}$ denotes the localisation of $\boldsymbol{Z}$ at $p$.

Also, $E_{n}^{2} \otimes \boldsymbol{Q}_{p}$ vanishes for $n>3, p=2$ and, we conjecture, for $p=3,5$ as well. Note that the 2-primary results are true by "overkill". The fact that we have multiplied by one more power of 2 than is necessary to kill the 2-primary part of $\pi_{*} S^{3}$ is enough to enable us to compute some (but unfortunately not all) of the extensions in the above sequence for $p=2$. In particular, we have $8 \mathrm{Sch}_{*} A_{5} \otimes Q_{2}=0$, and if $2 \pi_{j} S^{3} \otimes Q_{2}=0$ for $j=n-1$ or $n$, then the sequence above splits $(n>4, p=2)$. Thus, we have

$\operatorname{Sch}_{5} A_{5} \cong Z_{2} \oplus Z_{2}, \quad \operatorname{Sch}_{6} A_{5} \cong Z_{12} \oplus Z_{2}, \quad \operatorname{Sch}_{7} A_{5} \cong Z_{2} \oplus Z_{12}$, etc.

We have not yet found a nontrivial extension and suspect that every extension splits in the above sequence.

\section{REFERENCES}

1. D. Quillen, Higher K-theory for categories with exact sequences, Proc. 1972 Oxford Sympos., New Developments in Topology (to appear).

2. S. B. Priddy, Transfer, symmetric groups, and stable homotopy, Algebraic $K$ Theory. I: Higher $K$-Theories, Battelle Institute Conference, 1972, Lecture Notes in Math., vol. 341, Springer-Verlag, Berlin and New York, 1973, pp. 244-255.

3. H. S. M. Coxeter and W. O. J. Moser, Generators and relations for discrete groups, Springer-Verlag, Berlin 1957. MR 19, 527.

4. I. M. James, The suspension triad of a sphere, Ann. of Math. (2) 63 (1956), $407-$ 429. MR 18, 58.

5. H. Toda, On the double suspension $E^{2}$, J. Inst. Polytech. Osaka City Univ. Ser. A 7 (1956), 103-145. MR 19, 1188.

Department of Mathematics, University of Virginia, Charlottesville, VIRGINIA 22901 